\title{
Low Noise Amplifier Design for Ultra-WideBand Radio
}

\author{
Jongrit Lerdworatawee, Won Namgoong \\ Department of Electrical Engineering \\ University of Southern California \\ lerdwora@usc.edu,namgoong@usc.edu
}

\begin{abstract}
A new theoretical approach for designing a low-noise amplifier (LNA) for the ultra-wideband (UWB) radio is presented. Unlike narrowband systems, the use of the noise figure (NF) performance metric becomes problematic in UWB systems because of the difficulty in defining the signal-to-noise ratio (SNR). By defining the SNR as the matched filter bound (MFB), the NF measures the degree of degradation caused by the LNA in the achievable receiver performance after the digital decoding process. The optimum matching network that minimizes the NF as defined above has been solved. Since realizing the optimum matching network is in general difficult, an approach for designing a practical but suboptimum matching network is also presented. The NF performance of both the optimum and the suboptimum matching networks is studied as a function of the LNA gain.
\end{abstract}

\section{INTRODUCTION}

The ultra-wideband (UWB) radio is a relatively new technology that is being pursued for both commercial and military purposes [1][2]. It operates by spreading the energy of the radio signal very thinly over a wide bandwidth (e.g. several gigahertz). The rationale for deploying the UWB radio systems lies in the benefits of exceptionally wide bandwidths, thereby achieving a combination of very fine time/range resolution, ability to resolve multipath components, and favorable propagation condition of material penetration at low frequencies [3].

The goal of the receiver analog front-end is to condition the received analog signal for digitiziation, so that the highest performance can be achieved after decoding in the digital domain. The first and probably the most critical component of the analog frontend is the low noise amplifier (LNA), whose purpose is to amplify the received signal from the antenna with as little distortion and additional noise as possible. This is achieved by designing an appropriate matching network placed between the antenna and the amplifier.

This work was supported in part by the Army Research Office under contract number DAAD19-01-1-0477 and National Science Foundation under contract number ECS-0134629.
Unlike the narrowband LNA, the signal bandwidth of an UWB radio is several orders of magnitude greater. Hence, the underlying single tone signal assumption employed in narrowband LNA design becomes invalid, making many of the existing narrowband design techniques [4] based on this assumption also unsuitable.

The LNA is often designed to present an input impedence of $50 \Omega$ to avoid reflections on the transmission line connecting the off-chip antenna to the on-chip LNA. In this paper we consider a highly integrated UWB radio system. We assume that the antenna is placed in close proximity of the LNA, allowing us to ignore the $50 \Omega$ input impedence requirement.

For the noise factor (NF, or noise figure in $\mathrm{dB}$ ) of the LNA to be a meaningful metric in an UWB receiver, we define the SNR as the matched filter bound (MFB) [5], which represents an upper limit on the performance of data transmission systems. The MFB is obtained when a noise whitened matched filter is employed to receive a single transmitted pulse. By defining the SNR as the MFB, the NF measures the degree of degradation caused by the LNA in the achievable receiver performance after the eventual digital decoding process. In this paper, the optimum matching network that minimizes the NF as defined above has been solved. Since the optimal LNA matching network is generally difficult to realize in practice, we also present an approach for designing a sub-optimal but practical matching network.

The paper is organized as follows. The circuit and system model of the LNA-Antenna is presented in Section 2. In Section 3, the general solution to the optimal and suboptimal matching LNA are derived. Performance results are presented in Section 4. Conclusions are drawn in Section 5.

\section{CIRCUIT AND SYSTEM MODEL}

Throughout this paper, capital letters are used to denote the Fourier transforms (e.g. $X(\omega)$ ) of (voltage or current) system responses in the time domain, which are written in the corresponding lower case letters (e.g. $x(t)$ ). Sometimes the terms $\omega$ and $t$ are omitted for notational brevity unless needed for clarity.

\subsection{Circuit model of LNA-Antenna}

The quasi-static MOS transistor model is employed in this paper to account for the high-field effects in short-channel devices [6]. Accordingly, the transconductance $g_{m}$ and the gate-source 
capacitance $C_{g s}$ can be represented in terms of the power dissipation $P_{o}\left(=I_{d} V_{\text {supply }}\right)$ and the normalized gate overdrive $\rho\left(=\left(V_{g s^{-}}\right.\right.$ $\left.\left.V_{t h}\right) / L \varepsilon_{s a t}\right)$

$$
\begin{aligned}
g_{m} & =\frac{2 P_{o}}{V_{\text {supply }} L \varepsilon_{\text {sat }}}\left[\frac{1+\rho / 2}{\rho(1+\rho)}\right] \\
C_{g s} & =\frac{2}{3} \frac{P_{o}}{V_{\text {supply }} v_{\text {sat }} \varepsilon_{\text {sat }}}\left(\frac{1+\rho}{\rho^{2}}\right)
\end{aligned}
$$

where $L$ is the gate length, $V_{g s}$ is the gate-source bias voltage, $V_{t h}$ is the threshold voltage, $v_{\text {sat }}$ and $\varepsilon_{\text {sat }}$ are the saturation velocity and electric field, respectively.

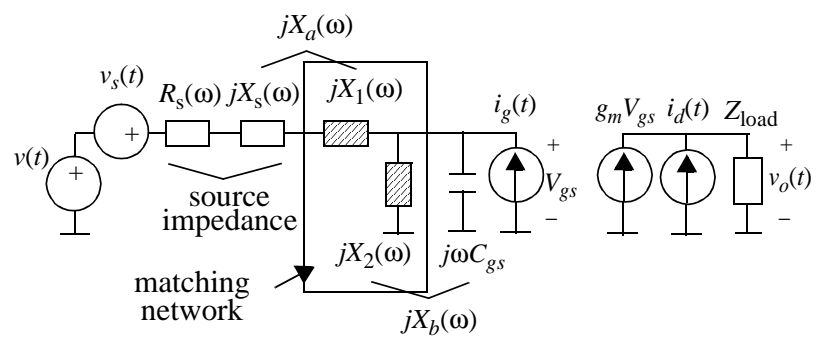

Figure 1 : Circuit model of LNA

Fig. 1 shows a circuit model of the analog front-end, including the antenna, the matching network, the LNA and a load, with three noise sources: the thermal voltage noise from the antenna resistance $v_{s}(t)$, the MOS gate current noise $i_{g}(t)$, and drain current noise $i_{d}(t)$. With no loss in generality, the antenna is modeled as a voltage source $v(t)$ with an impedance $Z_{s}(\omega)=R_{s}(\omega)+j X_{s}(\omega)$ while the amplifier is assumed as a common-source MOS transistor. The matching network is assumed lossless, consisting of two reactances, $X_{1}(\omega)$ and $X_{2}(\omega)$, as illustrated by the solid-line block in Fig. 1. For ease of analysis, the source reactance $X_{s}(\omega)$ is grouped with $X_{1}(\omega)$ and referred to as $X_{a}(\omega)$, and the gate-source capacitance $C_{g s}$ is grouped with $X_{2}(\omega)$ and referred to as $X_{b}(\omega)$.

The power spectral density (PSD) of the thermal noise from the antenna, the drain and the gate noise are given by

$$
\begin{gathered}
S_{v_{s}}(\omega)=4 k T R_{s}(\omega) \\
S_{i_{d}}(\omega)=4 k T \frac{\gamma}{\alpha} g_{m} \\
S_{i_{g}}(\omega)=4 k T \delta \alpha \frac{\left(\omega C_{g s}\right)^{2}}{5 g_{m}}
\end{gathered}
$$

where $k=1.38 \times 10^{-23} \mathrm{~J} / \mathrm{K}$ is the Boltzmann constant, $T$ is the absolute temperature, $\alpha$ is the ratio of $g_{m}$ to the zero-bias drain conductance, $\gamma$ and $\delta$ are the coefficients of channel and induced gate noise. Random noise process $i_{g}(t)$ is correlated to $i_{d}(t)$ with a correlation coefficient $c$ as given by [7]

$$
c=\frac{S_{i_{g} i_{d}}(\omega)}{\sqrt{S_{i_{g}}(\omega)} \cdot \sqrt{S_{i_{d}}(\omega)}}
$$

where $S_{i_{g} i_{d}}(\omega)$ is the PSD of the cross-correlation of $i_{g}(t)$ and $i_{d}(t)$. For a long channel device $c=0.395 j$. For lack of a more accurate value currently available, we assume that the long channel values for $c, \alpha, \gamma$ and $\delta$ are also valid in the short channel model employed in this paper.

\subsection{System model of LNA-Antenna}

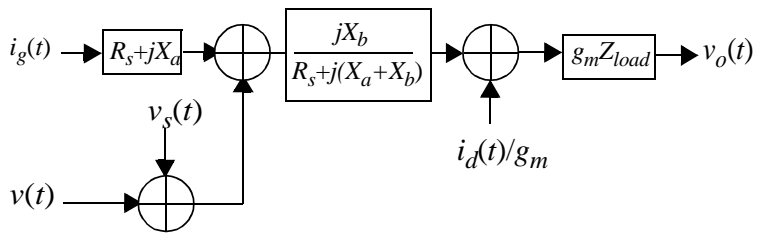

Figure 2 : System block diagram of LNA.

Fig. 2 is the system model of the circuit model in Fig. 1. The objective is to design the causal matching network (i.e., $X_{a}(\omega)$ and $\left.X_{b}(\omega)\right)$ so that the SNR at the output $v_{o}(t)$ is maximized. In the presence of $i_{g}(t)$, there exists an optimum gain in the matching network that balances the combined effects of $i_{g}(t), i_{d}(t)$ and signal amplification.

Note that $i_{g}(t)$ can be decomposed into two orthogonal components, i.e.

$$
i_{g}(t)=i_{g u}(t)+y_{c}(t) \otimes \frac{i_{d}(t)}{g_{m}}
$$

where $\otimes$ is the convolution operator, $i_{g u}(t)$ is the uncorrelated component of $i_{g}(t)$ to $i_{d}(t)$, and $y_{c}(t)$ is the equivalent correlation admittance between $i_{g}(t)$ and $i_{d}(t) / g_{m}$. From (4)-(7), the Fourier transform of $y_{c}(t)$ (i.e., $\left.Y_{c}(\omega)\right)$ can be obtained and given by

$$
\begin{aligned}
Y_{c}(\omega) & =g_{m} \frac{S_{i_{g} i_{d}}(\omega)}{S_{i_{d}}(\omega)}=j \omega C_{g s} \cdot\left\{\alpha|c| \sqrt{\frac{\delta}{5 \gamma}}\right\} \\
& \triangleq j X_{c}(\omega)
\end{aligned}
$$

Using the definition of the SNR described earlier, the SNR at the input of the LNA (i.e., the SNR of the received input signal) is given by [5]

$$
S N R_{i n}=\int \frac{A^{2}|P(\omega)|^{2}}{S_{v_{s}}(\omega)} d \omega
$$

where $P(\omega)$ is the normalized channel response to a single multipath component (i.e., $\|p\|^{2}=1$ ) and $A$ is the scaling factor of the received signal. With no loss in generality, we assume that the received signal is a 2 nd derivative of a Gaussian pulse [2].

Similarly, the SNR at the output of the LNA is

$$
S N R_{\text {out }}=\int \frac{A^{2}|P(\omega)|^{2}}{S_{n_{\text {out }}}(\omega)} d \omega
$$


where $S_{n_{\text {out }}}(\omega)$ represents the input-referred noise of all the noise sources in the LNA, which is given by

$$
\begin{aligned}
S_{n_{\text {out }}} & =S_{v_{s}}+S_{i_{\text {gu }}}\left(R_{s}^{2}+X_{a}^{2}\right) \\
& +\left[R_{s}^{2}\left(\frac{1}{X_{b}}-X_{c}\right)^{2}+\left(X_{a}\left(\frac{1}{X_{b}}-X_{c}\right)+1\right)^{2}\right] \frac{S_{i_{d}}}{g_{m}^{2}}
\end{aligned}
$$

where the spectrum of $i_{g u}(t)$ is

$$
S_{i_{g u}}(\omega)=4 k T \delta \alpha \frac{\left(\omega C_{g s}\right)^{2}}{5 g_{m}}\left(1-|c|^{2}\right)
$$

\section{LNA NOISE MATCHING}

\subsection{Optimal matching}

Reactances $X_{a}(\omega)$ and $X_{b}(\omega)$ that maximizes $S N R_{\text {out }}$ are obtained by differentiating (10) with respect to $X_{a}(\omega)$ and $X_{b}(\omega)$ and setting the result to zero. Assuming the output noise power of $i_{g u}(t)$ is less than that of $i_{d}(t)$ (i.e., $S_{i_{g u}}(\omega) R_{s}^{2}(\omega) g_{m}^{2}<S_{i_{d}}(\omega)$ ), which is typically the case, the optimum $X_{a}(\omega)$ and $X_{b}(\omega)$ (denoted as $X_{a, o p t}(\omega)$ and $\left.X_{b, o p t}(\omega)\right)$ can be solved:

$$
\begin{aligned}
& X_{a, \text { opt }}(\omega)= \pm \sqrt{R_{s}(\omega)\left(\sqrt{1 / \Gamma(\omega)}-R_{s}(\omega)\right)} \\
& X_{b, o p t}(\omega)=\frac{1}{X_{c}(\omega) \mp \sqrt{\frac{\Gamma(\omega)}{R_{s}(\omega)}\left(\sqrt{1 / \Gamma(\omega)}-R_{s}(\omega)\right)}}
\end{aligned}
$$

where $\Gamma(\omega)$ is

$$
\Gamma(\omega)=\frac{S_{i_{g u}}(\omega)}{S_{i_{d}}(\omega) / g_{m}^{2}}=\frac{\delta \alpha^{2}}{5 \gamma}\left(1-|c|^{2}\right)\left(\omega C_{g s}\right)^{2}
$$

Substituting (13) into (11), the optimum NF, denoted as $N F_{\text {opt }}$, is

$$
N F_{o p t}=\frac{\int|P(\omega)|^{2} d \omega}{\int \frac{|P(\omega)|^{2}}{1+\frac{\omega}{\omega_{T}} \sqrt{\frac{4 \delta \gamma}{5}\left(1-|c|^{2}\right)}} d \omega}
$$

where $\omega_{T}$ is the unity gain angular frequency:

$$
\omega_{T}=\frac{g_{m}}{C_{g s}}=\frac{3}{4} \frac{v_{\text {sat }}}{L} \frac{(1+\rho / 2) \rho}{(1+\rho)^{2}}
$$

Assuming a resistive load $Z_{\text {load }}\left(=R_{\text {load }}\right)$, the corresponding signal voltage power gain (in units of $\mathrm{V}^{2} / \mathrm{V}^{2}$ ) of the LNA, denoted as $G_{o p t}$, is given by

$$
G_{\text {opt }}=\frac{g_{m}^{2} R_{\text {load }}^{2} \int \frac{\left|X_{b}(\omega)\right|^{2}}{R_{s}^{2}+\left(X_{a, o p t}(\omega)+X_{b, o p t}(\omega)\right)^{2}}|P(\omega)|^{2} d \omega}{\int|P(\omega)|^{2} d \omega}
$$

As will be shown in the following sections, a trade-off between high $G_{o p t}$ and low $N F_{o p t}$ exists by varying $\rho$ for a given $P_{o}$.

Note that $X_{a, o p t}(\omega)$ and $X_{b, o p t}(\omega)$ given in (13) minimize the degradation in $S N R_{\text {out }}$ caused by the additive noise at every frequency, and hence they become independent of the received signal pulse. In a realistic matching network with a fixed structure, however, designing $X_{a}(\omega)$ and $X_{b}(\omega)$ with arbitrary reactances as assumed in the optimum matching network is in general not possible. The matching network then becomes a function of the transmitted signal pulse.

\subsection{Suboptimal matching}

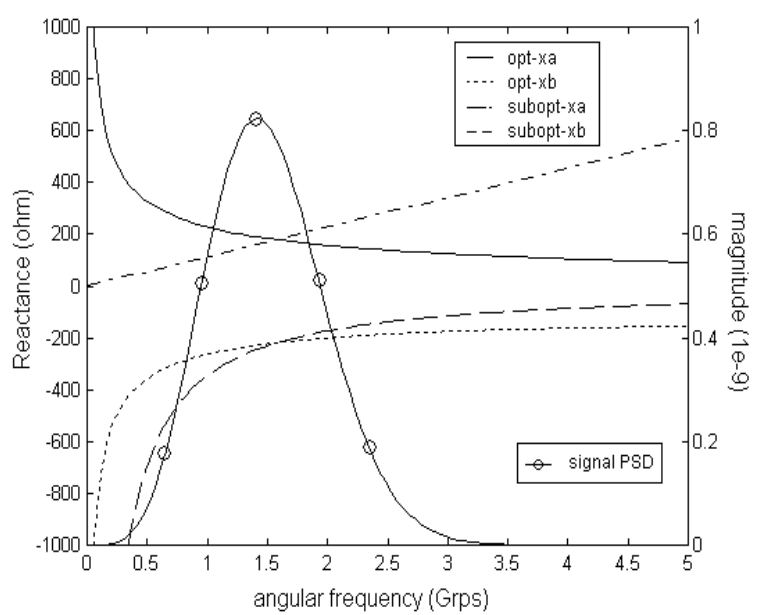

Figure 3 : suboptmal vs. optimal reactance in a signal region given $g_{m}=1 \mathrm{mS}$ and $C_{g s}=1 \mathrm{pF}$

Since realizing the optimum matching network is in general difficult, a heurisitc approach for determining a practical but suboptimum matching network is presented. Based on $X_{a, o p t}(\omega)$ and $X_{b, o p t}(\omega)$, a structure for the suboptimum matching network that best approximates the optimal response is first selected. The antenna impedance is assumed to be $50 \Omega$ across the bandwidth of interest. As shown in Fig. 3, the optimal matching network can be approximated by a two element $\left(L_{m}\right.$ and $\left.C_{m}\right)$ L-matching network, i.e., $X_{a}(\omega)=j \omega L_{a}$ and $X_{b}(\omega)=1 / j \omega C_{b}$, where $C_{b}=C_{g s}+C_{m}$ and $L_{a}$ $=L_{m}$. The choice of $L_{a}$ and $C_{b}$ is determined by numerically solving the following constrained optimization problem:

$$
\operatorname{minimize} N F=\frac{\int|P(\omega)|^{2} d \omega}{\int \frac{|P(\omega)|^{2}}{1+F_{1}(\omega)+F_{2}(\omega)} d \omega}
$$

subject to $L_{a} \geq 0, \quad C_{b} \geq C_{g s}$

where

$$
F_{1}(\omega)=\frac{\delta \alpha\left(\omega C_{g s}\right)^{2}}{5 g_{m} R_{s}}\left[R_{s}^{2}+\left(\omega L_{a}\right)^{2}\right]
$$




$$
\begin{aligned}
& F_{2}(\omega)=\frac{\gamma}{\alpha g_{m} R_{s}}\left\{R_{s}^{2} \omega^{2}\left[C_{b}+C_{g s}\left(\alpha|c| \sqrt{\frac{\delta}{5 \gamma}}\right)\right]^{2}\right. \\
& +\left[1-\omega^{2} L_{a} C_{b}\left(C_{b}+\omega C_{g s}\left(\alpha|c| \sqrt{\frac{\delta}{5 \gamma}}\right)\right]^{2}\right\}
\end{aligned}
$$

The cost function (i.e., NF) in (18) is obtained by substituting $X_{a}(\omega)$ and $X_{b}(\omega)$ of the L-matching network into (10) and (11). Similar to (17), the corresponding signal voltage power gain (in units of $V^{2} / V^{2}$ ) is

$$
G=\frac{g_{m}^{2} R_{\text {load }}^{2} \int \frac{|P(\omega)|^{2}}{\left(1-\omega^{2} L_{a} C_{b}\right)^{2}+\left(\omega R_{s} C_{b}\right)^{2}} d \omega}{\int|P(\omega)|^{2} d \omega}
$$

\section{PERFORMANCE RESULTS}

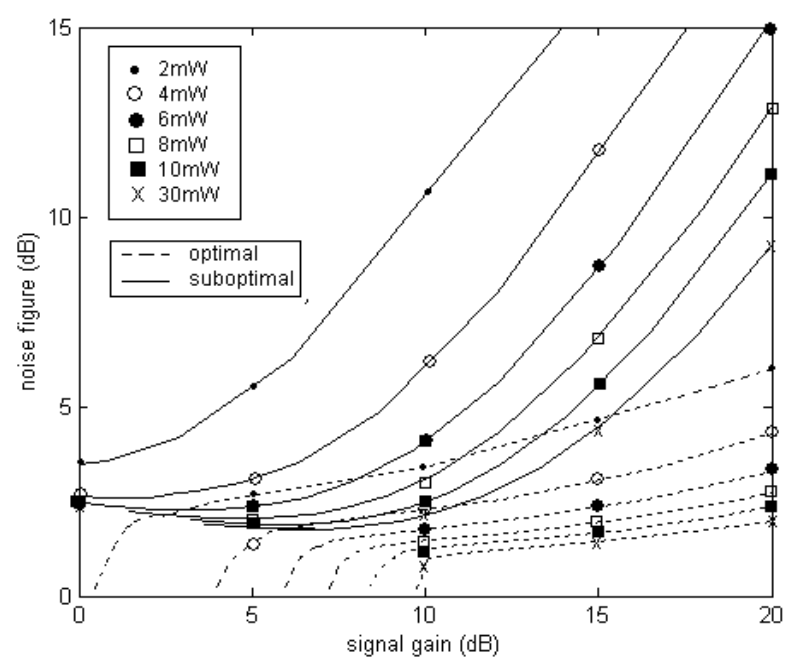

Figure 4 : Contours of noise figure and signal gain relating $\rho$ for a specified $P_{o}$ (the number on the curves)

In Fig. 4 the NF is plotted against the signal voltage gain $\mathrm{G}$ (in units of $\mathrm{V}^{2} / \mathrm{V}^{2}$ ) for both the optimum (in dash-line) and the suboptimum matching networks (in solid-line) when $P_{o}$ is fixed. In the optimum matching network, a trade-off between reducing $\mathrm{NF}$ and increasing $\mathrm{G}$ can be made by varying the normalized gate overdrive $\rho$. For sufficiently high $G$, large increases in $\mathrm{G}$ causes only a small increase in NF. For example, increasing $\mathrm{G}$ from 10 $\mathrm{dB}$ to $20 \mathrm{~dB}$ when $P_{o}$ is $10 \mathrm{~mW}$ increases the NF by less than 1 $\mathrm{dB}$. In the suboptimum matching network, there is an optimum $\mathrm{G}$ that minimizes the NF. For example, when $P_{o}$ is $10 \mathrm{~mW}$, the optimum $\mathrm{G}$ is approximately $7 \mathrm{~dB}$. If operating below the optimum $\mathrm{G}$, the NF does not increase much. However, if $G$ is increased beyond the optimum point, the NF increases abruptly. For example, an increase in $\mathrm{G}$ from $10 \mathrm{~dB}$ to $20 \mathrm{~dB}$ when $P_{o}$ is $10 \mathrm{~mW}$ increases the NF by almost $10 \mathrm{~dB}$. Hence, the LNA that dissipates $P_{o}$ should not be designed to operate with a gain that is much greater than the optimum G. Another important observation is that for a fixed $\mathrm{NF}$, increasing $\mathrm{G}$ by increasing $P_{o}$ suffers from diminishing returns. For example, given a target NF of approximately 3
$\mathrm{dB}$, increasing $P_{o}$ by $20 \mathrm{~mW}$ from $10 \mathrm{~mW}$ to $30 \mathrm{~mW}$ improves $\mathrm{G}$ by less than $1.5 \mathrm{~dB}$; whereas increasing $P_{o}$ by only $6 \mathrm{~mW}$ from 4 $\mathrm{mW}$ to $10 \mathrm{~mW}$ increases $\mathrm{G}$ by more than $11 \mathrm{~dB}$. This diminishing returns in $\mathrm{G}$ suggests that large signal amplification is most efficiently achieved in multiple stages.

\section{CONCLUSIONS}

A generalized approach for designing an UWB LNA that minimizes the NF with the SNR defined using the MFB has been developed. The matching network consists of two lossless reactances, which are connected in series and in parallel to the MOS amplifier. The optimum matching network depends only on the LNA device noise while the suboptimum matching network depends also on the received signal and noise. This additional dependency of the suboptimum matching network results because designing matching networks with arbitrary reactances as assumed in the optimum matching network is in general not possible.

For a simple LC suboptimum matching network that we considered, there exists an optimum $\mathrm{G}$ that minimizes the NF for a given power dissipation. Although the optimum $\mathrm{G}$ can be increased by increasing power consumption, this approach suffers from diminishing returns. Hence, a single stage amplification may not be sufficient; more complex matching network or multiple amplification stages with attendant complexity may be required.

\section{REFERENCES}

[1] M. Z. Win and R. A. Scholtz, "Impulse radio: How it works," IEEE Commun. Lett., vol. 2, no. 2, pp.36-38, Feb. 1998.

[2] M. Z. Win and R. A. Scholtz, "Ultra-wide bandwidth timehopping spread-spectrum impulse radio for wireless multiaccess communications," IEEE Trans. Commnu., vol. 48, no. 4 , pp. $679-691$, Apr. 2000

[3] M. Z. Win and R. A. Scholtz, "On the roubustness of ultrawide bandwidth signals in dense multipath environments," IEEE Commun. Lett., vol. 2, no. 2, pp. 5153, Feb. 1998.

[4] D. K. Shaeffer, T. H. Lee, "A 1.5-V, 1.5-Hz CMOS low noise amplifier," IEEE J. Solid-State Circuits, vol. 32, pp. 745-759, May. 1997.

[5] J. Cioffi, "EE379A Course Notes," Stanford University.

[6] K.-Y. Toh, P.-K. Ko, and R. G. Meyer, "An Engineering model for short-channel mos devices," IEEE J. Solid-Sate Circuits, vol. 23, no. 4, pp. 950-958, Aug. 1988.

[7] D. P. Triantis, A. N. Birbas, and D. Kondis, "Thermal noise modeling for short-channel MOSFET's," IEEE Trans. Electron Devices, vol. 43, pp. 1950-1955, Nov. 1996. 